\title{
Energy Efficiency and Reliability Tradeoff in Optical Core Networks
}

\author{
Pawel Wiatr, Jiajia Chen, Paolo Monti, Lena Wosinska \\ KTH Royal Institute of Technology, School of ICT, Optical Networks Lab, Electrum 229, 16440 Kista, Sweden \\ Email:wosinska@kth.se
}

\begin{abstract}
We assess the highest allowable reliability performance degradation of active components caused by applying energy-efficient mechanisms. EDFAs are identified as the most critical devices where energy saving might not cover the potential additional reparation cost.

OCIS codes: (060.0060) Fiber optics and optical communications; (060.4250) Networks
\end{abstract}

\section{Introduction}

There are many research activities aiming at reducing the energy consumption within the ICT sector, with a particular attention to telecommunication networks [1][2]. Most of these green approaches are based on the concept that network components that are not used are either switched off or put into a low power state (sleep mode) [3]. However, frequent transitions between operational and sleep/off conditions may negatively impact the component reliability performance [4]. For example, cyclic temperature changes cause faster deterioration of solder connections [5][6], thus potentially reducing the circuit's life time. This possible reliability performance degradation may introduce additional operational expenditures (OPEX) in terms of failure reparation, which has to be taken into account in the overall OPEX calculation when energy saving techniques are used in a network. Therefore, a tradeoff between the energy (and consequently the cost) saved by a specific green strategy and the extra expenses deriving from a shorter lifetime of one or more network components would need to be considered in some cases. This tradeoff can be measured in terms of maximum allowable failure rate increase where the extra reparation cost due to the additional failures would be covered by the saving obtained by a given green strategy. An assessment in this direction was done for optical access networks [4], where it was found that the frequent state transitions caused by a sleep-mode-based energy-efficient scheme may even increase the overall OPEX in the network. On the other hand, even though a number of green provisioning strategies have been proposed for optical core networks (e.g., [7][8]) none of them analyze the possible impact these strategies have on the network components' life time.

The aim of the current work is to understand the importance of this impact for the operational cost in optical core networks. With this objective in mind, we first identify the components that are the most vulnerable to the reliability performance degradation. It is found that erbium doped fiber amplifiers (EDFAs) can tolerate the lowest failure rate increase among all the considered active network devices. This also implies that green routing and wavelength assignment (RWA) algorithms leveraging on EDFAs in sleep mode could severely affect the overall network OPEX. Therefore, the second part of the paper presents a case study, where the potential energy consumption benefits deriving from one specific power aware RWA strategy [7] are assessed against the additional reparation cost caused by the potential failure rate increase of the EDFAs.

\section{Maximum allowable failure rate increase: a component level assessment}

This section presents an assessment of the maximum allowable failure rate increase of the main active components used in a wavelength division multiplexing (WDM) core network. The analysis is done by considering a number of potential energy saving thresholds, as a result of different energy saving profiles.

The total operational expenditure $O P E X_{T}$ related to a given component is defined as a sum of energy consumption and reparation related costs and is calculated based the following quantities:

- $O P E X_{E} \quad$ - the cost related to energy consumption;

- $\triangle O P E X_{E}$ - the energy savings obtained by a low power mode operation;

- $O P E X_{F} \quad$ - the reparation cost in normal operating conditions;

- $\triangle O P E X_{F}$ - the cost increase related to the additional failure reparation(s) caused by the increased failure rate as a consequence of the transitions between low and high power modes.

Other operational expenses (e.g., floor space renting, building maintenance, service provisioning costs) are not considered because they do not change whether or not a low power mode is used. As a result $O P E X_{T}=O P E X_{E}-$ $\triangle O P E X_{E}+O P E X_{F}+\triangle O P E X_{F}$. The OPEX $X_{E}$ and $\triangle O P E X_{E}$ consider both the energy cost (USD $/ \mathrm{kWh}$ ) and the device energy consumption in active and sleep mode. The $O P E X_{F}$ and $\triangle O P E X_{F}$ take into account failure rate, failure rate variation, failure reparation cost (as a function of manpower cost), Mean Time To Repair (MTTR), and number of personnel required for reparation. The details of the OPEX models are presented in [4][9]. The respective OPEX and $\triangle$ OPEX costs are calculated in a per year basis. We define the maximum allowable failure rate increase for a given 
component when energy related cost saving compensates the additional failure related cost, i.e., $\Delta \mathrm{OPEX}_{\mathrm{E}}=$ $\triangle \mathrm{OPEX}_{\mathrm{F}}$.

Table 1 shows how the maximum allowable failure rate increase varies (in percent) as a function of a number of energy saving thresholds. Results are presented for different active network components. Passive components such as splitters, (de)multiplexers, and patch panels are not considered in this study because they are not the target of any energy saving mechanism. For each component the table also provides information about failure rate, MTTR, number of personnel required for failure reparation (i.e., Pers.), and energy consumption, all under normal operation conditions. The failure rate is measured in FIT (Failure in Time), which corresponds to one failure during $10^{9}$ hours. The energy price and the labor rate are assumed to be $0.27 \mathrm{USD} / \mathrm{kWh}$ and $190 \mathrm{USD} / \mathrm{h}$, respectively [4].

Table 1. Maximum allowable failure rate increase as a function of possible energy savings.

\begin{tabular}{|c|c|c|c|c|c|c|c|c|c|c|c|}
\hline \multirow{2}{*}{ Component } & \multirow{2}{*}{$\begin{array}{c}\text { Failure } \\
\text { rate [FIT] }\end{array}$} & \multirow{2}{*}{$\begin{array}{c}\text { MTTR } \\
{[\mathrm{h}]}\end{array}$} & \multirow{2}{*}{ Pers. } & \multirow{2}{*}{$\begin{array}{c}P \\
{[W]}\end{array}$} & \multicolumn{7}{|c|}{ Max. allowable failure rate increase with energy saving of: } \\
\hline & & & & & $5 \%$ & $10 \%$ & $25 \%$ & $50 \%$ & $75 \%$ & $90 \%$ & $95 \%$ \\
\hline Transponder [9] & 256 & 2 & 1 & 70 & $947.5 \%$ & $1895 \%$ & $4737 \%$ & $9475 \%$ & $14213 \%$ & $17056 \%$ & $18004 \%$ \\
\hline Regenerator [9] & 256 & 2 & 1 & 70 & $947.5 \%$ & $1895 \%$ & $4737 \%$ & $9475 \%$ & $14213 \%$ & $17056 \%$ & $18004 \%$ \\
\hline Optical Switch [10] & 5467 & 2 & 1 & 60 & $38.0 \%$ & $76.1 \%$ & $190.1 \%$ & $380.3 \%$ & $570.5 \%$ & $684.6 \%$ & $722.6 \%$ \\
\hline $\begin{array}{l}\text { Reconfigurable Optical Add/Drop } \\
\text { Multiplexer (ROADM) [11] }\end{array}$ & 3300 & 2 & 1 & 35 & $36.8 \%$ & $73.5 \%$ & $183.8 \%$ & $367.5 \%$ & $551.3 \%$ & $661.5 \%$ & $698.3 \%$ \\
\hline EDFA [9] & 2000 & 6 & 2 & 8 & $2.3 \%$ & $4.6 \%$ & $11.6 \%$ & $23.1 \%$ & $34.7 \%$ & $41.6 \%$ & $43.9 \%$ \\
\hline
\end{tabular}

As shown in Table 1, the maximum allowable failure rate increase of transponders/regenerators may vary between 948\% and 18004\% (depending on the energy profile). For Reconfigurable Optical Add/Drop Multiplexers (ROADMs) and optical switches the maximum allowable failure rate increase is smaller than the one of transponders/regenerators, but still considerably high. As a result, it can be concluded that for these components the impact of frequent on/sleep transitions on the reliability performance is not a concern. In fact the extra reparation cost is easily compensated by the energy savings, i.e., $\triangle \mathrm{OPEX}_{\mathrm{E}}>>\Delta \mathrm{OPEX}_{\mathrm{F}}$.

However, for EDFAs the maximum allowable failure rate increase is relatively small, i.e., between $2.3 \%$ and 43.9\%. Even when the energy savings might be potentially high (i.e., 95\%) the maximum allowable failure rate increase is only $43.8 \%$. As of today, there are no available data to assess the impact that on/off transitions have on the failure rate of currently deployed EDFAs. It is, on the other hand, possible to speculate around these values using data available for other electronic devices. After all, the underling physical phenomena responsible for the decrease of the reliability performance are similar (e.g., temperature variation causing strain creation in the solder [5],[6]). The study in [12] shows that when a sleep-mode-based approach is applied to a System on Chip (SoC), the failure rate increases between $11 \%$ and $43 \%$ for energy savings levels between $50 \%$ and $90 \%$, respectively. If a similar reliability performance decrease is assumed for EDFAs then, based on the results in Table 1, there might be instances in which using energy saving strategies may not be beneficial anymore, i.e., $\Delta \mathrm{OPEX} E \Delta \mathrm{OPEX}$. This has to be assessed case by case because both $\triangle \mathrm{OPEX}_{\mathrm{E}}$ and $\triangle \mathrm{OPEX}_{\mathrm{F}}$ depend on a number of factors including the specific network topology and the energy saving strategy in use. In the next section we carry out a case study on EDFA for a specific network scenario and elaborate the maximum allowable reliability performance decrease by putting the amplifiers in sleep mode.

\section{Case study}

This section presents a case study where the maximum allowable failure rate increase and the potential energy saving obtained using the Weighted Power Aware - Lightpath Routing (WPA-LR) algorithm [7] is assessed in a European backbone network, i.e., COST 239.

In general, power aware RWA schemes try to minimize the network energy consumption by maximizing the number of unused fiber links so that their respective EDFAs can be put in sleep mode. The WPA-LR algorithm uses the same intuition. More specifically WPA-LR bases its RWA decisions on whether or not a given fiber link is already used to carry the traffic. If a fiber link is not in use, its routing cost is set to the power necessary to operate all the EDFAs deployed along its length (i.e., its energy consumption). If, on the other hand, a fiber link is in use its routing cost becomes the product of the fiber link power and a parameter $\alpha$ that varies in a range [0;1]. Values of $\alpha$ close to 0 , encourage WPA-LR to select routes at minimum energy cost, while with $0<\alpha<1$ WPA-LR tends to make routing choices that are a compromise between energy consumption minimization and (fiber) resource efficiency maximization. More details on the WPA-LR strategy, and the COST 239 network parameters are available in [7]. This specific case study is based on the link state information (average number of active/sleeping links for a given value of the network load) gathered as a result of the WPA-LR strategy, and it uses them to calculate the achievable 
energy savings (focusing only on EDFAs contribution). These energy savings results are then used and to assess the maximum allowable failure rate increase for the EDFAs in the network.

Figure 1 presents these values as a function of $\alpha$ and the network load. As explained before with $\alpha=1$ WPA-LR behaves as Shortest Path routing algorithm with First Fit wavelength assignment. The maximum allowable failure rate increase is relatively low and it decreases rapidly with the increasing load values (i.e., when there are lower chances to save energy by putting EDFAs to sleep). On the other hand, with values of $\alpha$ close to 0 higher values of the maximum allowable failure rate increase can be observed. Regardless of the value of $\alpha$, the observed maximum allowable failure rate increase is well within the range typical for SoC, confirming our expectation mentioned in the previous section. Table 2 presents the relative OPEX savings/losses, i.e., $\left(\triangle \mathrm{OPEX}_{\mathrm{E}}-\Delta \mathrm{OPEX}_{\mathrm{F}}\right) / \mathrm{OPEX}_{\mathrm{T}}$ as a function of network load and the failure rate increase, when $\alpha=0.66$. This value of $\alpha$ provides a reasonable compromise between energy savings and efficient use of the network resources [7]. As expected the savings are getting smaller when the failure rate increases, reaching negative values (losses) when the failure rate increase is higher than the maximum allowable level. It can also be observed that the overall OPEX savings decrease quite rapidly. The reason is twofold: first, EDFAs have the highest reparation cost among all the considered components, e.g., due to the technicians travel-related expenses, and second, the potential energy savings are low as EDFAs are low power devices. Overall, these considerations make EDFAs critical elements to be considered in energy saving techniques.

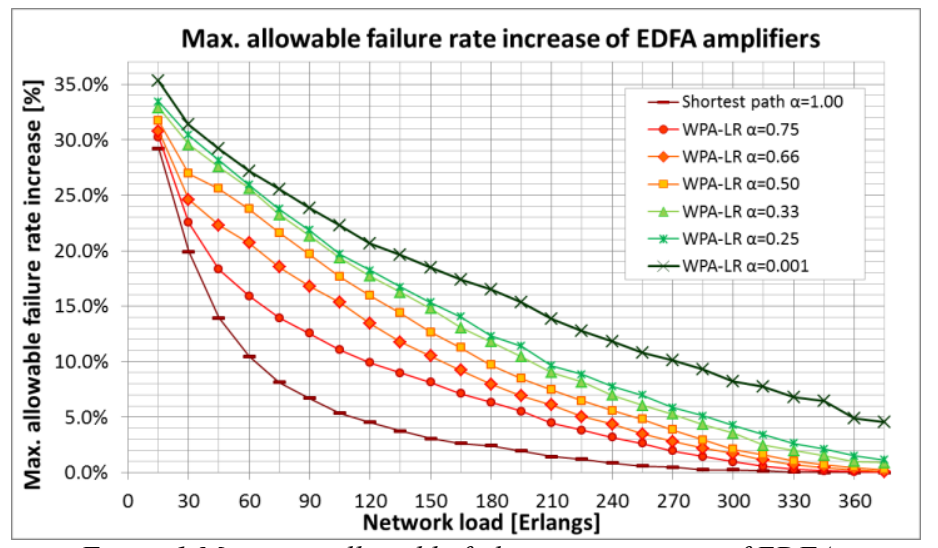

Table 2 Relative OPEX savings/losses (in percentage) in function of the load and (possible) failure rate increase.

\begin{tabular}{|c|c|c|c|c|c|}
\hline \multirow{2}{*}{$\begin{array}{c}\text { Load } \\
\text { [Erlangs] }\end{array}$} & \multicolumn{5}{|c|}{ Failure rate increase [\%] } \\
\hline & $\mathbf{0 \%}$ & $5 \%$ & $10 \%$ & $15 \%$ & $20 \%$ \\
\hline 0 & $32.1 \%$ & $28.8 \%$ & $25.4 \%$ & $22.0 \%$ & $18.6 \%$ \\
\hline 15 & $20.9 \%$ & $17.5 \%$ & $14.1 \%$ & $10.7 \%$ & $7.3 \%$ \\
\hline 60 & $14.1 \%$ & $10.7 \%$ & $7.3 \%$ & $3.9 \%$ & $0.5 \%$ \\
\hline 120 & $9.1 \%$ & $5.7 \%$ & $2.3 \%$ & $-1.1 \%$ & $-4.5 \%$ \\
\hline 240 & $3.0 \%$ & $-0.4 \%$ & $-3.8 \%$ & $-7.2 \%$ & $-10.6 \%$ \\
\hline 375 & $0.1 \%$ & $-3.3 \%$ & $-6.7 \%$ & $-10.1 \%$ & $-13.5 \%$ \\
\hline
\end{tabular}

Figure 1 Maximum allowable failure rate increase of EDFA.

\section{Conclusions}

There are many promising green approaches proposed to save energy in optical core networks. However, their possible negative impact on the reliability performance should be taken into account, and the related additional fault management cost should be assessed. This paper analyzes the tradeoff between energy savings and reliability performance degradation for different active components in optical core networks. The results show that EDFAs are critical in this respect, since the benefits form energy saving that they can offer may easily be overtaken by the additional reparation cost. Consequently, the green RWA schemes based on putting EDFAs in a sleep mode may not be beneficial from the OPEX perspective.

\section{Acknowledgement}

The research leading to these results has received funding from the European Community's Seventh Framework Programme (FP7/2007-2013) under grant agreement $\mathrm{n}^{\circ} 318137$ ICT-DISCUS.

\section{References}

[1] Ch. Lange et al., "Energy Consumption of Telecommunication Networks and Related Improvement Options, ” IEEE Journal of Selected Topics in Quantum Electronics, March/Apr. 2011.

[2] K. Hinton et al., "Power consumption and energy efficiency in the internet," IEEE Network, March/Apr. 2011.

[3] Z. Yi et al., "Energy Efficiency in Telecom Optical Networks," IEEE Communications Surveys \& Tutorials, Q4 2010.

[4] P. Wiatr et al., "Energy saving in access networks: Gain or loss from the cost perspective?, " ICTON 2013, June 2013.

[5] W. Engelmeier, "Solder Joints in Electronics: Design for Reliability".

[6] K. Setty et al., "Powercycling Reliability, Failure Analysis and Acceleration Factors of Pb-Free Solder Joints, " $55^{\text {th }}$ Conference on Electronic Components and Technology, May/June 2005.

[7] P. Wiatr et al., "Power savings versus network performance in dynamically provisioned WDM networks, "IEEE Commun. Mag., May 2012.

[8] A. Coiro et al. "Power-Aware Routing and Wavelength Assignment in Multi-Fiber Optical Networks," IEEE/OSA JOCN, Nov. 2011.

[9] A. Leiva et al., "Upgrading cost modeling of capacity-exhausted static WDM networks," ONDM 2012, April 2012.

[10] Optical Access Seamless Evolution (OASE), FP7 project, http://www.ict-oase.eu/.

[11] The DIStributed Core for unlimited bandwidth supply for all Users and Services (DISCUSs), FP7 project, http://www.discus-fp7.eu/.

[12] T.S. Rosing et al., "Power and Reliability Management of SoCs," IEEE Transactions on VLSI Systems, Apr. 2007. 